\title{
Guest Editorial: Test and Verification Challenges for Future Microprocessors and SoC Designs
}

\author{
Sandip Ray • Jay Bhadra • Magdy S. Abadir • \\ Li-C Wang
}

Received: 7 September 2013 / Accepted: 7 September 2013 / Published online: 10 October 2013

(C) Springer Science+Business Media New York 2013

With increasing sophistication of VLSI technology, process, and architecture, microprocessors and SoC systems continue to increase in complexity. This has resulted in an increasing trend in design errors, manufacturing flaws, and security holes in modern VLSI systems. As computing moves from servers, desktops, and laptops to datacenters and clouds on the one hand, and phones, tablets, and wearables on the other, the situation is expected to be exacerbated with increasingly diverse form factors, high integration complexity, and more complex manufacturing process. Consequently, verification and testing technology will continue to dominate as crucial factors in time-tomarket, reliability, and cost of VLSI systems. There has been significant research on verification and testing chal-

\author{
S. Ray $(\square)$ \\ Strategic CAD Labs, Intel Corporation, \\ Hillsboro, OR 97124, USA \\ e-mail: sandip.ray@intel.com \\ J. Bhadra $\cdot$ M. S. Abadir \\ Technology Solutions Organization, \\ Freescale Semiconductor Inc., \\ Austin, TX 78729, USA \\ J. Bhadra \\ e-mail: jayanta.bhadra@freescale.com \\ M.S. Abadir \\ e-mail: m.abadir@freescale.com \\ L.-C. Wang \\ Department of Electrical and Computer Engineering, \\ University of California at Santa Barbara, \\ Santa Barbara, CA 93106, USA \\ L.-C. Wang \\ e-mail: licwang@ece.ucsb.edu
}

lenges and their synergy for modern and future VLSI systems. However, much of the research is scattered across different conference proceedings and journal issues, and entail different (and often conflicting) approaches, formalisms, and assumptions. It is time to provide a focused and unified look at the domain, identify and consolidate the key problems and emerging trends in test and verification, and reflect on how to apply the techniques synergistically for ensuring reliability of current and future systems.

This special issue documents some recent progress in this challenging research area. Note that this is a vast area with a large diversity of interesting research topics ranging from tools, techniques, methodologies, and target applications. Covering the entire gamut of this research of course, is beyond the scope of a single special issue. Instead, our goal has been to provide a sampling of the different facets of this exciting domain, and highlight some key emerging trends. With that goal in mind, we have picked seven representative articles covering several orthogonal research directions in the broad spectrum of verification and testing challenges the next-generation computing. Many of the papers in this issue are extended versions of work presented at the Microprocessor Test and Verification Workshop (MTV) 2012. Indeed, the special issue was inspired by consistent contributions to the area by MTV participants over the last decade, and in particular by several significant presentations on the topic in MTV 2012. However, the issue includes other papers as well. Each paper was rigorously peer-reviewed to ensure that it meets the journal standards. Since a key goal is to provide a comprehensive reference for the different research challenges and progress, each article includes a detailed discussion of related research. 
The first paper, "Observation-Oriented ATPG and Scan Chain Disabling for Capture Power Reduction" by Lung-Jen Lee, presents a method for reducing test power for testing integrated circuits. The method combines test pattern generation with scan chain disabling to achieve low capture power, and is shown to be empirically efficient.

The second paper, "LLVMVF: A Generic Approach for Verification of Multicore Software" by Sousa and Sen, addresses another important challenge in verification, viz., concurrency. With computing going to multicore, there is a critical need today to ensure that (concurrent) software running on multicore systems operate correctly. The paper presents a new framework called LLVM Verification Framework LLVMF that provides a scalable approach for verification of multicore software. The framework targets Low Level Virtual Machine (LLVM) byte-code representation of multicore software, and the paper discusses the use of the framework on verification of multicore software using Pthreads library.

The third paper, "On the Reuse of Heterogeneous IPs into SysML Models for Integration Validation" by Bombieri et al. addresses two important issues in the verification of SoC designs: integration validation and IP reuse. A critical factor in SoC design is efficient, accurate integration of IP components; however, this raises the challenge of quickly validating the integration. The approach described in the paper targets integration validation of SysML models. In particular, it discusses an approach for efficient reuse of already existing heterogeneous IPs into a SysML models during integration validation, without requiring the designer to generate SysML abstractions for those IPs.

The next two papers deal with challenges involving transaction-level models (TLMs). With growing complexity of hardware designs, transaction-level models are getting increasingly viewed as an important abstraction that can act as a manageable verification target while providing meaningful correlation to lower-level (RTL or netlist) implementation. On the other hand, TLMs come with their own verification challenges. In "Automatic Generation of System Level Assertions from Transaction Level Models", Liu and Vasudevan discuss an important challenge, generation of assertions. Assertion generation is critical, since they define the specifications used as verification targets; thus, the more complete and accurate the assertions the better the quality of results of verification. The paper discusses how to use machine learning techniques on simulation traces to generate high-quality assertions for
TLM designs, and offers experimental results demonstrating the scalability and accuracy of the method. Next, in "A Semantics-based Translation Method for Automated Verification of SystemC TLM Designs", Gao and Il discuss an approach to translate TLM designs into a formal model which can then be analyzed formally using existing model checkers.

The final two papers deal with another interesting emerging area, verification of asynchronous and analog systems. Modern systems increasingly have analog/mixedsignal (AMS) components integrated together with a number of digital blocks; furthermore, global synchronization is being increasingly difficult, thus making asynchronous, self-timed system designs a potentially attractive alternative. Uygur and Sattler, in "A Novel Formalism for Partially Defined Asynchronously Feed-Backed Digital Circuits", look at an important aspect of asynchronous systems, viz., partial definition due to metastability. They develop a formalism for such partially defined asynchronously feedbacked digital circuit, and how the formalism can handle metastability and analog feedback in digital structures. Finally, in "Formal Verification of Analog and Mixed Signal Designs using SPICE Circuit Simulation Traces", Lata and Roy present an approach for verification of analog/mixed-signal designs. They use analog simulations from SPICE to formally verify AMS blocks, and provide a technique for hierarchical verification of the system using simulation results from different abstraction levels of the design.

The future of computing will likely include a large diversity of systems and platforms, with unique challenges in verification and testing. Development of effective, scalable test and verification technologies for all the different platforms will likely remain an elusive goal in the foreseeable future. However, the area is vast, the challenge is real, and there is encouraging trend in attacking designs in different domains with generic but domain-specific theories, tools, and methodologies. Perhaps through a crosspollination of these efforts we will one day successfully address the test and verification challenges of future computing systems. Of course, a single journal issue can only scratch the surface of the area. We hope that these seven articles can provide a source for further references and research.

We express our sincere thanks to all the authors and referees for their contribution in creating this special issue. We thank Prof. Vishwani Agrawal (EIC, JETTA), for his 
encouragement and support, and Maria Rhea Perilla for technical help in this long process. We hope you enjoy this issue and that it inspires more research to overcome further challenges in test and verification of future systems.

Sandip Ray is a Research Scientist at Strategic CAD Labs, Intel Corporation. His current research focus is on developing post-silicon validation infrastructure for next-generation microprocessor and SoC designs. In particular, he leads projects on observability constraints and metrics for post-silicon debug quality, and techniques for post-silicon test readiness. Before joining Intel, Sandip worked as a Research Scientist at University of Texas at Austin, where he developed formal verification techniques for mathematical analysis of diverse computing systems ranging from synthesized hardware designs to Java programs. Sandip is the author of one book, as well as more than 30 peer-reviewed research articles on system validation and verification techniques. He has served on the program committee of more than 20 international meetings and conferences, as co-chair for the International Conference on Formal Methods in Computer-Aided Design (FMCAD 2013) and International Workshop on the ACL2 Theorem Prover and Its Applications (ACL2 2009), and as a guest editor for ACM Transactions on Design Automation of Electronic Systems. Sandip has a Ph.D in Computer Science from the University of Texas at Austin. He is a senior member of IEEE and a professional member of ACM.

Jay Bhadra is currently the World-wide technical lead and senior engineering manager for Freescale Semiconductor's Verification/Validation related CAD Tools, Flows \& Methodologies Central team. Jay received the Ph.D. in Computer Engineering from the University of Texas at Austin and has worked on digital hardware verification through formal/semi-formal techniques. Jay is a senior member of the IEEE and taught Verification as an Adjunct Associate Professor of Computer Engineering at University of Texas at Austin. Jay has co-authored 50+ technical papers, received two international best paper awards, and filed several issued/pending U.S. patents.
Magdy S. Abadir has a B.S. with honors in Computer Science from the University of Alexandria, Egypt, an M.S. in Computer Science from the University of Saskatchewan, Saskatoon, Canada, and a Ph.D. degree in Electrical Engineering from the University of Southern California, Los Angeles. Currently he is the Director of Design Automation, Technology Solutions Organization, Freescale Semiconductor. Prior to joining Freescale (Motorola) he was the General Manager of Best IC Labs in Austin Texas. From 1986 to 1994 he worked at the Microelectronics and Computer Technology Corporation in various leadership roles and he reached the level of senior member of the technical staff. Dr. Abadir has been selected in 2005 as an IEEE fellow for contribution the verification and test of microprocessors. He has 10 patents issued, in addition to several that have been filed. He co-founded and chaired a series of international workshops on the economics of design, test and manufacturing and on microprocessor test and verification (MTV). He has co-edited several books on those subjects, and also published over 250 technical journal and conference papers in the areas of test economics, design for test, and design verification and economics. Four of his papers received best paper awards. Dr. Abadir also served as an adjunct faculty at the University of Texas at Austin.

Li-C Wang is Professor in the ECE department at University of California, Santa Barbara. He received Ph.D. in 1996 from University of Texas at Austin. He was a senior CAD software technical staff member at Somerset PowerPC Design Center, Motorola from 1996 to 2000. Dr. Wang received best paper awards from DATE-1998, IEEE VTS1999, and DATE-2003. He is the recipient of the Technical Excellence Award from Semiconductor Research Cooperation (SRC) in 2010 for his contribution in bringing data mining technologies into SRC member companies. He co-founded the IEEE Microprocessor Test and Verification (MTV) Workshop, and is currently the program chair. $\mathrm{He}$ is currently serving or had served as technical PC member for workshops and conferences including ITC, VTS, ICCAD, DATE, ISQED, HLDVT, ITSW, DATA, ATS, ICCD, VLSI-DAT, etc and is currently serving as the general co-chair for VLSI-DAT. He is an associate editor of IEEE Transactions on CAD and also guest editor of a number of D\&T special issues. Since 2004, he has published more than 60 papers on the topics of applying data mining and machine learning in test, verification, and validation. 